\title{
CONUT Score or/and Peripheral Blood CD4+/CD8+ Ratio-Based Web Dynamic Nomograms to Predict the Individualized Survival of Patients with Advanced Osteosarcoma
}

This article was published in the following Dove Press journal:

Cancer Management and Research

\author{
Qian-Kun Yang $\mathbb{D}^{1, *}$ \\ Yan-Na Su ${ }^{2, *}$ \\ Wei Wang ${ }^{1, *}$ \\ Nan Wang ${ }^{3}$ \\ Zhong-Xiang Yao ${ }^{4, *}$ \\ Xiao-Jing Zhang'
}

'Department of Bone and Soft Tissue Surgery, Cancer Hospital of China Medical University, Liaoning Cancer Hospital and Institute, Shenyang, Liaoning I I0042, People's Republic of China; ${ }^{2}$ Clinical Laboratory, The First Affiliated Hospital of China Medical University, Shenyang, Liaoning I 10042, People's Republic of China; ${ }^{3}$ Department of Radiotherapy, Cancer Hospital of China Medical University, Liaoning Cancer Hospital and Institute, Shenyang, Liaoning I I0042, People's Republic of China; ${ }^{4}$ Department of Physiology, Army Medical University, Chongqing 400038, People's Republic of China

*These authors contributed equally to this work
Correspondence: Xiao-Jing Zhang Department of Bone and Soft Tissue Surgery, Cancer Hospital of China Medical University, Liaoning Cancer Hospital and Institute, Shenyang, Liaoning I 10042, People's Republic of China Email zhangxiaojing@cancerhosp-ln-cmu. com
Background: Nutritional and immune status is paramount for the overall survival (OS) of patients with advanced osteosarcoma. Comprehensive prognostic predictors based on the two indices are scarce. This study aimed to construct and validate individualized web dynamic nomograms based on CONUT score or/and peripheral blood CD4+/CD8+ ratio for OS in patients with advanced osteosarcoma.

Materials and Methods: The clinical data of 376 advanced osteosarcoma patients from January 2000 to December 2019 were retrospectively collected. Data from the 301 patients (diagnosed in the first 15 years) were used as the development set and data from the remaining 75 patients were assigned as the validation set. Multivariate Cox regression analyses were conducted and three prediction models were constructed, namely, CD4 +/CD8+ ratio univariate model (model 1), CONUT score univariate model (model 2), and CD4+/CD8+ ratio plus CONUT score (model 3). These models were visualized by conventional nomograms and individualized web dynamic nomograms, and their performances were further evaluated by $\mathrm{C}$-index, calibration curve, receiver operating characteristic (ROC) curve, and decision curve analysis (DCA), respectively.

Results: In multivariate Cox analysis, age, metastasis, ALP, CD4+/CD8+ ratio, chemotherapy, and CONUT score were identified as independent prognostic factors for OS. The calibration curves of the three models all showed good agreement between the actual observation and nomogram prediction for 1-year overall survival. In the development set, the $\mathrm{C}$-index and area under the curve (AUC) of model $3(0.837,0.848)$ were higher than that of model $1(0.765,0.773)$ and model $2(0.712,0.749)$. Similar trends were observed in the validation set. The net benefits of model 3 were better than the other two models within the threshold probability of $36-80 \%$ in DCA.

Conclusion: CONUT score and peripheral $\mathrm{CD} 4+/ \mathrm{CD} 8+$ ratio are easily available, reliable, and economical prognostic predictors for survival prediction and stratification in patients with advanced osteosarcoma, but the two predictors combined can establish a better prognosis prediction model.

Keywords: CONUT score, osteosarcoma, survival, nomogram

\section{Introduction}

Osteosarcoma is a highly malignant mesenchymal tumor that primarily occurred in bone and characterized by adolescent onset, obvious disease heterogeneity, and high susceptibility of early lung metastasis. ${ }^{1}$ The development and utilization of modern 
multidisciplinary therapy (MDT) have significantly improved the prognosis of osteosarcoma, and the 5-year overall survival (OS) rate has significantly increased from less than $20 \%$ to approximately $70 \%$ in patients with nonmetastatic disease. ${ }^{2,3}$ However, regarding patients with metastatic osteosarcoma, not all the patients can benefit from MDT due to the heterogeneity of the disease, and the prognosis was still dismal, with the 5-year survival rate being less than $30 \% .{ }^{4}$ One of the primary obstacles for this failure was the lack of effective and reliable prognostic predictive biomarkers to guide pre-treatment risk stratification and treatment protocol selection.

A series of factors have revealed their predictive or prognostic values in multiple malignancies and they can be roughly categorized into two types: traditionally established prognostic factors and newly-identified predictive factors. Clinicopathologic features, inflammatory indices, nutritional indices, immune indices, and metabolic indices were the most commonly used and conventionally established prognostic indicators. ${ }^{5-10}$ In contrast, some differentially expressed and tissue-specific proteins, microRNAs (miRNAs), long non-coding RNAs (lncRNAs), and circular RNAs (circRNAs) can also serve as reliable prognosticators in various neoplasms. ${ }^{11-14}$ It is undoubtedly that all these predictors inherently and ineluctably harbor their merits and drawbacks. Generally, traditionally established predictors are more accessible and affordable than the newly-identified prognosticators, but they cannot rival the latter in predictive specificity and accuracy. Therefore, how to identify an ideal prognostic predictor is a hard nut pending to be conquered. An economic and pragmatic approach to circumvent such dilemma is to establish new prognostic predictors with the currently identified prognosticators.

For patients with advanced malignant diseases, nutritional and immune status is the most pivotal prognostic factors for survival. ${ }^{15,16} \mathrm{~A}$ favorable nutritional condition is the prerequisite to perform any therapeutic regimen, such as chemotherapy, radiotherapy, or targeted therapy. Malnourished patients usually cannot withstand these treatments and thus are more prone to obtain worse outcomes. Actually, malnutrition or undernourishment is an already identified prognosticator for unfavorable outcomes in various malignancies, including osteosarcoma. ${ }^{17-20}$ The Controlling Nutritional Status (CONUT) score, calculated from serum albumin, total cholesterol, and total lymphocyte counts (TLC), is an objective nutrition scoring system that has been extensively utilized to assess nutritional status in various malignant diseases, such as renal cell carcinoma, hepatocellular carcinoma, and gastric cancer. $^{21-24}$ A notable advantage of this scoring system is that all the data for its constituent parameters can be readily calculated from routine biochemical or blood tests, and their dynamic changes can be easily monitored by clinicians. Unfortunately, although CONUT score is a reliable prognosticator for various cancers, its prognostic and predictive significance in osteosarcoma remains undetermined.

Immune function, another vital prognosticator for advanced neoplasms, participates in multiple biological processes of tumors, including tumorigenesis, progression, and metastasis. Numerous immune predictors have exhibited their prognostic values in cancers, including absolute lymphocyte count (ALC), platelet-lymphocyte ratio (PLR), lymphocyte-monocyte ratio (LMR), neutrophillymphocyte ratio (NLR), peripheral blood lymphocyte subsets analysis (PBLSA), tumor-infiltrating lymphocytes (TILs), tumor-associated macrophages (TAMs), and other immune infiltrations in the tumor microenvironment (TME). ${ }^{25-34}$ Regarding osteosarcoma, higher NLR or PLR, and lower LMR or ALC were significantly associated with inferior outcomes. ${ }^{25-27}$ Besides, pre-treatment intratumoral $\mathrm{CD} 8+$ /FOXP3+-ratio $\leq 3.08$, decreased circulating CD8+ $\mathrm{T}$ cells to T-regulatory cells (Tregs) (CD8 +/Tregs) ratio, or increased percentage of peripheral blood CD4+CXCR5 $+\mathrm{T}$ cells usually portend unfavorable survival or metastasis, while early recovery of peripheral blood lymphocytes during chemotherapy is associated with better prognosis in osteosarcoma. ${ }^{1,28,35}$ Among all these immune prognostic predictors, PBLSA is the most convenient and pragmatic prognosticator for tumors. Especially, CD4+/CD8+ ratio, a commonly used parameter of PBLSA, has already revealed its prognostic values in various tumors, including laryngeal cancer, renal cell carcinoma, melanoma, and breast cancer. ${ }^{36-39}$ However, similar to CONUT score, the clinical significance of CD4 +/CD8+ ratio in osteosarcoma also remains uncertain.

Given the paramount role of immunity and nutrition, as well as the potent prognostic values of CONUT score and $\mathrm{CD} 4+/ \mathrm{CD} 8+$ ratio in other malignancies, it is reasonable to speculate that prognostic systems based on them may harvest optimal and reliable predictive performances in osteosarcoma. Therefore, our study aimed to study the predictive and prognostic values of CONUT score or/and peripheral blood CD4+/CD8+ ratio-based prediction models in patients with advanced osteosarcoma. 


\section{Patients and Methods}

\section{Patient Selection and Study Design}

This retrospective observational study was scrutinized and approved by the ethical committee of Cancer Hospital of China Medical University (also named Liaoning Cancer Hospital and Institute). The requirement for informed consent was waived in this study due to the nature of retrospective design and anonymization of the data. By reviewing the electronic medical records, the clinical data of 376 advanced osteosarcoma patients admitted to the department of bone and soft tissue surgery from January 2000 to December 2019 were retrospectively collected. Inclusion criteria of participants were as follows: (i) pathologically confirmed as osteosarcoma; (ii) the existence of at least one metastatic lesion beyond the primary tumor site determined by the image examination or with late stage disease; and (iii) with detailed and completed extractable medical data and were not lost to follow-up. Patients who met the following criteria were excluded from the study: (i) any history of other malignancies; (ii) pulmonary nodules or metastatic lesions derived from other malignant diseases; and (iii) incomplete or unextractable medical data and laboratory results.

In this study, 301 patients from January 2000 to December 2014 were utilized as the development set, while the remaining 75 patients from January 2014 to December 2019 were assigned as the validation set.

\section{CONUT Score Definition and Peripheral Blood Lymphocyte Subsets Analysis}

CONUT score is a comprehensive nutritional and immune index calculated from serum albumin, serum total cholesterol concentration, and peripheral TLC, as previously reported. ${ }^{40}$ The three constituent parameters of CONUT score were scored based on the cut-off values reported in previous studies, ${ }^{41-43}$ and the sum of the score was the CONUT score. Patients were stratified into four groups according to the CONUT score listed in Table 1.

Peripheral whole blood samples were taken before breakfast in the morning and collected into heparinized tubes for PBLSA. Before the conduction of flow cytometric analysis, a series of treatments for heparinized blood were performed, including mixed with fluorescencelabeled monoclonal antibodies, incubated for 30 minutes, washed by phosphate-buffered saline (PBS), centrifuged at $1500 \mathrm{rpm}$ for 5 minutes, discarded the supernatant and resuspended the remaining pellet. Finally, an eligible
Table I The Details of CONUT Score

\begin{tabular}{|c|c|c|c|c|}
\hline \multirow{2}{*}{\begin{tabular}{|l} 
Parameters \\
$\begin{array}{l}\text { Serum } \\
\text { albumin }(g / d L)\end{array}$
\end{tabular}} & \multicolumn{4}{|c|}{ Range (Score) } \\
\hline & $\geq 3.5$ (I) & $3.0-3.49(2)$ & $2.5-2.9(4)$ & $<2.5(6)$ \\
\hline $\begin{array}{l}\text { Serum total } \\
\text { cholesterol } \\
(\mathrm{mg} / \mathrm{dL})\end{array}$ & $\geq 180(0)$ & $140-180(1)$ & $100-139(2)$ & $<100(3)$ \\
\hline $\begin{array}{l}\text { Lymphocyte } \\
\text { count }\left(/ \mathrm{mm}^{3}\right)\end{array}$ & $\geq 1600(0)$ & $1200-1599$ (I) & $800-1199$ (2) & $<800(3)$ \\
\hline $\begin{array}{l}\text { CONUT } \\
\text { score }\end{array}$ & $0-1$ & $2-4$ & $5-8$ & $9-12$ \\
\hline $\begin{array}{l}\text { Nutrition } \\
\text { stratification }\end{array}$ & Normal & Mild & Moderate & Severe \\
\hline
\end{tabular}

Abbreviation: CONUT score, controlling nutritional status score.

sample was obtained for flow cytometric analysis. The examinations for each patient were performed three times and the average of the three outcomes was used for final statistical analysis. The detailed steps for PBLSA can refer to the descriptions in relevant literature. ${ }^{44-46}$

\section{Data Collection}

The baseline demographics and variables listed below were obtained at diagnosis, including age, gender, NLR, PLR, metastasis, primary tumor site, tumor size, alkaline phosphatase (ALP), anti-angiogenesis therapy, peripheral blood CD4 +/CD8+ ratio, chemotherapy, and CONUT score.

\section{Follow-Up}

The follow-up started when the patients were diagnosed with advanced, late stage, or metastatic disease, and it ended until death or December 2019. The end-point for this study was overall survival (OS), which can be calculated from the date of the initial diagnosis of advanced or metastatic disease to death (event) from any cause or the last date of follow-up (censored).

\section{Statistical Analyses}

\section{Patient Characteristics and Continuous Variable Grouping}

The Shapiro-Wilk test was conducted to determine the normality of the measurement data. The continuous variables were presented as median (interquartile range) while the categorical variables were presented as frequencies (percentages).

Comparisons of clinical characteristics between the development set and validation set were conducted by Fisher's exact test or chi-squared test for categorical data, and Wilcoxon's rank-sum test for measurement data. 
For the continuous variables of age, NLR, PLR, ALP and $\mathrm{CD} 4+/ \mathrm{CD} 8+$ ratio, their optimal cut-off points were identified by the method of the receiver operating characteristic (ROC) curve analyses based on the maximal Youden's index. These variables were then dichotomized into two groups based on the optimal cut-off points. The survival differences between groups for the variables of CD4+/CD8+ ratio and CONUT score were examined by the Kaplan-Meier survival curve and Log-rank test.

\section{Model Construction and Visualization}

The Cox proportional hazards model and the stepwise selection method based on minimal Akaike information criterion (AIC) were used to determine the final predictors from the potential variables. The Schoenfeld residual plots and variance inflation factor (VIF) were also performed to examine the Cox proportional hazards model assumption and multicollinearity of variables, respectively. Based on the results from Cox analyses, three predictive models (Model 1-3) and nomograms to predict median survival time (MST), 1-year survival probability, and 3-year survival probability were constructed. Furthermore, the three predictive models were visualized in two ways, including conventional nomograms and the newly developed interactive web dynamic nomograms.

\section{Model Evaluation}

After the establishment of the nomograms, their predictive performances were evaluated, including discrimination and calibration. Discrimination represents the ability to distinguish survivors from non-survivors at specific time nodes, which is often evaluated by plotting the ROC curve and then calculating and comparing its related parameters, such as the area under curve (AUC). Therefore, we firstly drew three ROC curves at the time point of 1-year overall survival and then calculated its related evaluating indices of the three models in the development and validation cohort, including AUC, sensitivity (Sen), specificity (Spe), positive predictive value $(\mathrm{PV}+)$, negative predictive value (PV-), positive likelihood ratio $(\mathrm{LR}+)$, and negative likelihood ratio (LR-). Moreover, the Harrell's concordance index (C-index) was also calculated and compared among the three models in both sets.

The calibration curves were also plotted to assess the concordance between the predicted survival and observed survival. Furthermore, decision curve analysis (DCA) was utilized to compare the performance and superiority of the three predictive models. Besides, survival differences in the entire cohort based on quartile groupings of the total predictive scores calculated from the three established prediction models were also compared.

\section{Software}

All statistical analysis was performed by R software (version 3.6.0) with the assistance of several essential $\mathrm{R}$ packages, including rms, foreign, nomogramEx, stdca. $\mathrm{R}$, survival, survminer, survivalROC, pROC, dplyr, pec, devtools, DynNom, shiny, rsconnect, and ggplot2 package. A two-tailed $P$-value lower than 0.05 was considered as statistically significant.

\section{Results}

\section{Patient Characteristics}

The ROC curves for variables of age, NLR, PLR, ALP, $\mathrm{CD} 4+/ \mathrm{CD} 8+$ ratio, and CONUT score are presented in Figure 1. The optimal cut-off points for the first five continuous variables were $20,3.4,171,154$, and 3.27 , respectively. The clinical characteristics of the development and validation set are presented in Table 2. A total of 376 advanced osteosarcoma patients were finally enrolled in this study, including 301 patients in the development set and 75 patients in the validation set. In the development set, the median follow-up time was 8.9 months (range: $0.5-90.1$ months) and the median survival time was 8.1 months (95\% CI: 6.1-10.7 months). In the validation set, the median follow-up time and the median survival time were 8.6 months (range: 0.5-88.7 months) and 8.2 months (95\% CI: 5.7-11.6 months), respectively. No significant differences were found among all the variables between the development set and validation set (all $P>0.05)$.

\section{Identification of Prognostic Factors, Construction, and Visualization of Predictive Models}

The results of univariate and multivariate Cox regression analysis for overall survival are shown in Table 3. In the univariate Cox regression analyses, variables of age $(P=0.022)$, gender $(P=0.027)$, NLR $(P=0.033)$, metastasis $(P=0.000)$, ALP $(P=0.000)$, anti-angiogenesis therapy $(P=0.000), \mathrm{CD} 4+/ \mathrm{CD} 8+$ ratio $(P=0.000)$, chemotherapy $(P=0.000)$ and CONUT score $(P=0.002)$ were significantly associated with OS in the development set. The KaplanMeier analyses and Log-rank tests for $\mathrm{CD} 4+/ \mathrm{CD} 8+$ ratio and CONUT score also confirmed the results from 
A

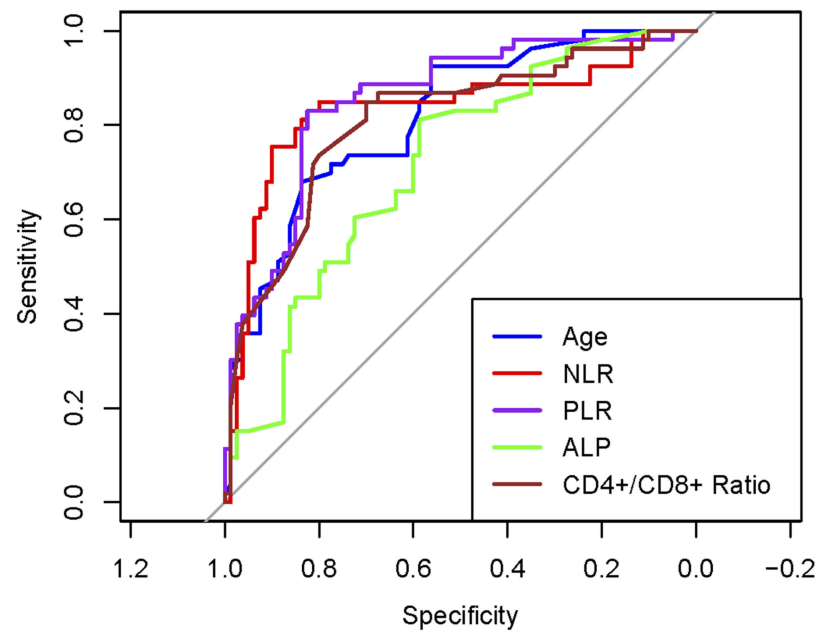

B

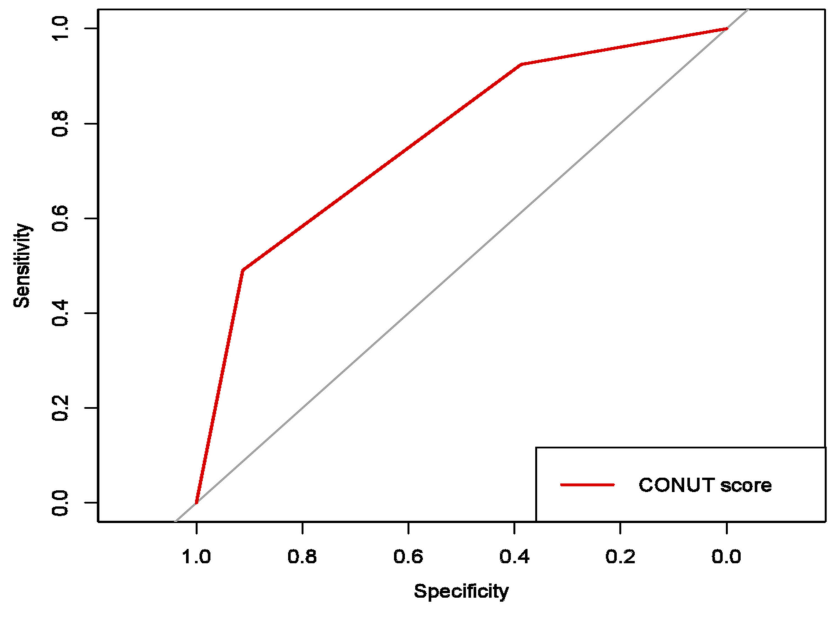

Figure I The receiver operating characteristic (ROC) curve analyses for (A) age, NLR, PLR, ALP, CD4+/CD8+ ratio, and (B) CONUT score.

Abbreviations: NLR, neutrophil to lymphocyte ratio; PLR, platelet to lymphocyte ratio; ALP, alkaline phosphatase; CD, cluster of differentiation; CONUT score, controlling nutritional status score.

univariate Cox analysis. The survival curves of the two variables are shown in Figure 2. Regarding multivariate Cox regression analyses, age $(P=0.000)$, metastasis $(P=0.000)$, ALP $(P=0.004), \mathrm{CD} 4+/ \mathrm{CD} 8+$ ratio $(P=0.000)$, chemotherapy $(P=0.001)$ and CONUT score $(P=0.000)$ were confirmed as independent prognostic factors for OS.

Three prediction models were established to predict the MST, 1-year, and 3-year survival probability in patients with advanced osteosarcoma. The details of the parameters of the three models are presented in Table 4, and the detailed point for each predictor included in the prediction models is shown in Table 5. Furthermore, the variables used to establish prediction models together with websites for web dynamic nomograms are presented in Table 6. In this study, two methods were used to visualize the three predictive models, including conventional nomograms and the newly developed interactive web dynamic nomograms. The conventional nomograms are presented in Figure 3, and the websites for interactive web dynamic nomograms are shown in Table 6.

\section{Prediction Model Validation and Performance Assessment}

Calibration curves to predict the 1-year OS probability in both sets are plotted and shown in Figure 4. All calibration curves showed good consistency between the actual survival and nomogram predicted survival. ROC curves and its related parameters were also performed to further validate the discrimination ability of three prediction models at the time point of 1-year OS. The ROC curves are shown in
Figure 5 and the parameters reflecting the accuracy of ROC curves are presented in Table 7.

In the development set, the $\mathrm{C}$-indexes of model 1 , model 2 and model 3 were 0.765 [95\% CI, 0.712-0.828], 0.712 [95\% CI, 0.738-0.908] and 0.837 [95\% CI, 0.735-0.908], respectively, and significant differences were found among the three models (model 1 vs model 2, $P=0.043$; model 3 vs model $1, P=0.015$; model 3 vs model $2, P=0.003$ ). In the validation set, the $\mathrm{C}$-indexes of the three models were 0.771 [95\% CI, 0.654-0.827], 0.723 [95\% CI, 0.667-0.876] and 0.841 [95\% CI, 0.766-0.927], respectively. Similar trends in the differences of $\mathrm{C}$-indexes were also found for the three models (model 1 vs model 2, $P=0.047$; model 3 vs model 1, $P=0.023$; model 3 vs model $2, P=0.004$ ).

\section{Decision Curve Analysis}

Figure 6 illustrates the decision curve analyses of three prediction models to discriminate survivors and nonsurvivors at the time point of 1-year OS. All models remained effective and reliable between the threshold probabilities of $36-80 \%$, and the net benefit for model 3 was better than that of the other two models within the same threshold probabilities.

\section{Kaplan-Meier Survival Analysis Based on Risk Stratification}

The entire cohort was categorized into four groups based on the quartiles of total prognostic scores calculated from the three predicting models. The Kaplan-Meier analyses 
Table 2 Baseline Characteristics of the Development Set and Validation Set

\begin{tabular}{|c|c|c|c|c|c|}
\hline Variables & $\begin{array}{l}\text { Total } \\
(n=376)\end{array}$ & $\begin{array}{l}\text { Development Set } \\
(n=301)\end{array}$ & $\begin{array}{l}\text { Validation Set } \\
(n=75)\end{array}$ & Statistics & $P$ value \\
\hline $\begin{array}{l}\text { Age } \\
\quad<20 \text { years } \\
\geq 20 \text { years }\end{array}$ & $\begin{array}{l}173(46.0 \%) \\
203(54.0 \%)\end{array}$ & $\begin{array}{l}138(45.8 \%) \\
163(54.2 \%)\end{array}$ & $\begin{array}{l}35(46.7 \%) \\
40(53.3 \%)\end{array}$ & 0.016 & 0.899 \\
\hline $\begin{array}{l}\text { Gender } \\
\text { Male } \\
\text { Female }\end{array}$ & $\begin{array}{l}236(62.8 \%) \\
140(37.2 \%)\end{array}$ & $\begin{array}{l}192(63.8 \%) \\
109(36.2 \%)\end{array}$ & $\begin{array}{l}44(58.7 \%) \\
3 I(4 I .3 \%)\end{array}$ & 0.674 & 0.412 \\
\hline $\begin{array}{l}\text { NLR } \\
\quad<3.4 \\
\geq 3.4\end{array}$ & $\begin{array}{l}120(31.9 \%) \\
256(68.1 \%)\end{array}$ & $\begin{array}{l}94(31.2 \%) \\
207(68.8 \%)\end{array}$ & $\begin{array}{l}26(34.7 \%) \\
49(65.3 \%)\end{array}$ & 0.326 & 0.568 \\
\hline $\begin{array}{l}\text { PLR } \\
\quad<|7| \\
\geq|7|\end{array}$ & $\begin{array}{l}369(98.1 \%) \\
7(1.9 \%)\end{array}$ & $\begin{array}{l}294(97.7 \%) \\
7(2.3 \%)\end{array}$ & $\begin{array}{l}75(100.0 \%) \\
0(0.0 \%)\end{array}$ & 1.777 & 0.182 \\
\hline $\begin{array}{l}\text { Metastasis } \\
\text { Lung only } \\
\text { Lung and other sites }\end{array}$ & $\begin{array}{l}152(40.4 \%) \\
224(59.6 \%)\end{array}$ & $\begin{array}{l}|2|(40.2 \%) \\
180(59.8 \%)\end{array}$ & $\begin{array}{l}3 I(4 I .3 \%) \\
44(58.7 \%)\end{array}$ & 0.032 & 0.858 \\
\hline $\begin{array}{l}\text { Tumor site } \\
\text { Axial skeleton } \\
\text { Extremity } \\
\text { Tumor size (median/interquartile } \\
\text { range, cm) } \\
\text { ALP } \\
\text { Normal }(<154 \mathrm{IU} / \mathrm{L}) \\
\text { Elevated }(\geq 154 \mathrm{IU} / \mathrm{L})\end{array}$ & $\begin{array}{l}217(57.7 \%) \\
159(42.3 \%) \\
18.0(5.4-28.8) \\
315(83.8 \%) \\
61(16.2 \%)\end{array}$ & $\begin{array}{l}179(59.5 \%) \\
122(40.5 \%) \\
18.3(5.4-28.8) \\
255(84.7 \%) \\
46(15.3 \%)\end{array}$ & $\begin{array}{l}38(50.7 \%) \\
37(49.3 \%) \\
17.1(5.7-28.2) \\
60(80.0 \%) \\
15(20.0 \%)\end{array}$ & $\begin{array}{l}1.906 \\
0.000 \\
0.983\end{array}$ & $\begin{array}{l}0.167 \\
0.996 \\
0.321\end{array}$ \\
\hline $\begin{array}{l}\text { Anti-angiogenesis therapy } \\
\text { No } \\
\text { Yes }\end{array}$ & $\begin{array}{l}334(88.8 \%) \\
42(11.2 \%)\end{array}$ & $\begin{array}{l}270(89.7 \%) \\
31(10.3 \%)\end{array}$ & $\begin{array}{l}64(85.3 \%) \\
\mathrm{II}(14.7 \%)\end{array}$ & 1.154 & 0.283 \\
\hline $\begin{array}{l}\text { CD4+/CD8+ ratio } \\
\text { High }(\geq 3.27) \\
\text { Low }(<3.27)\end{array}$ & $\begin{array}{l}315(83.8 \%) \\
61(16.2 \%)\end{array}$ & $\begin{array}{l}249(82.7 \%) \\
52(17.3 \%)\end{array}$ & $\begin{array}{l}66(88.0 \%) \\
9(12.0 \%)\end{array}$ & 1.230 & 0.267 \\
\hline $\begin{array}{l}\text { Chemotherapy } \\
\geq 6 \text { cycles } \\
<6 \text { cycles }\end{array}$ & $\begin{array}{l}70(18.6 \%) \\
306(81.4 \%)\end{array}$ & $\begin{array}{l}59(19.6 \%) \\
242(80.4 \%)\end{array}$ & $\begin{array}{l}\text { II(14.7\%) } \\
64(85.3 \%)\end{array}$ & 0.965 & 0.326 \\
\hline $\begin{array}{l}\text { CONUT score } \\
\text { Normal } \\
\text { Mild+moderate } \\
\text { Severe }\end{array}$ & $\begin{array}{l}17(4.5 \%) \\
233(62.0 \%) \\
126(33.5 \%)\end{array}$ & $\begin{array}{l}\mid 4(4.7 \%) \\
\mid 86(6 \mid .8 \%) \\
10 \mid(33.6 \%)\end{array}$ & $\begin{array}{l}3(4.0 \%) \\
47(62.7 \%) \\
25(33.3 \%)\end{array}$ & 0.065 & 0.968 \\
\hline
\end{tabular}

Abbreviations: NLR, neutrophil to lymphocyte ratio; PLR, platelet to lymphocyte ratio; ALP, alkaline phosphatase; CD, cluster of differentiation; CONUT score, controlling nutritional status score.

and Log-rank tests were performed to compare the differences in OS among the four groups. Figure 7 presents the Kaplan-Meier survival curves for the three models based on quartile groupings. Significant differences in survival were found among the four subgroups in the three models (all $P<0.001$ ). However, survival curves of model 3 separate more significantly than those from the other two models, indicating a better discrimination ability.

\section{Discussion}

The present study is the first to elucidate the relationship between CONUT score and peripheral 
Table 3 Univariate and Multivariate Cox Regression Analyses for Overall Survival in the Development Set

\begin{tabular}{|c|c|c|c|c|c|c|}
\hline \multirow[t]{2}{*}{ Variables } & \multicolumn{3}{|c|}{ Univariate Analysis } & \multicolumn{3}{|c|}{ Multivariate Analysis } \\
\hline & HR & $95 \% \mathrm{Cl}$ & $P$ value & HR & $95 \% \mathrm{Cl}$ & $P$ value \\
\hline Age & 1.258 & $1.037-1.592$ & 0.022 & 1.523 & $1.217-1.907$ & 0.000 \\
\hline Gender & 0.781 & $0.627-0.973$ & 0.027 & 0.930 & $1.205-1.509$ & 0.768 \\
\hline NLR & 1.283 & $1.021-1.611$ & 0.033 & 1.219 & $0.732-2.030$ & 0.446 \\
\hline PLR & 0.809 & $0.334-1.957$ & 0.638 & 0.793 & $0.319-1.973$ & 0.618 \\
\hline Metastasis & 2.184 & $1.749-2.728$ & 0.000 & 2.537 & $2.002-3.214$ & 0.000 \\
\hline Tumor site & 0.903 & $0.729-1.120$ & 0.354 & 0.917 & $0.727-1.158$ & 0.469 \\
\hline Tumor size & 1.006 & $0.985-1.028$ & 0.561 & 1.020 & $0.998-1.042$ & 0.082 \\
\hline ALP & 1.950 & $1.465-2.594$ & 0.000 & 1.787 & $1.200-2.662$ & 0.004 \\
\hline Anti-angiogenesis therapy & 2.260 & $1.619-3.154$ & 0.000 & 1.103 & $0.696-1.748$ & 0.675 \\
\hline CD4+/CD8+ ratio & 3.132 & $2.347-4.178$ & 0.000 & 3.008 & $2.218-4.080$ & 0.000 \\
\hline Chemotherapy & 1.734 & $1.308-2.299$ & 0.000 & $1.66 \mathrm{I}$ & $1.245-2.216$ & 0.001 \\
\hline CONUT score & & & 0.002 & & & 0.000 \\
\hline Normal & Ref & Ref & & Ref & Ref & \\
\hline Mild+moderate & 1.186 & $0.722-1.950$ & 0.501 & 1.657 & $0.994-2.76 \mid$ & 0.053 \\
\hline Severe & 1.788 & $1.068-2.994$ & 0.027 & 2.597 & I.527-4.417 & 0.000 \\
\hline
\end{tabular}

Abbreviations: NLR, neutrophil to lymphocyte ratio; PLR, platelet to lymphocyte ratio; ALP, alkaline phosphatase; CD, cluster of differentiation; HR, hazard ratio; Ref, reference; CONUT Score, controlling nutritional status score.

blood CD4+/CD8+ ratio and survival in patients with advanced osteosarcoma. We found that higher CONUT score and lower peripheral blood CD4+/CD8+ ratio was associated with shorter OS and increased risks of death. Both of the two indices can serve as prognostic predictors for OS, but the combined use of them can obtain better predictive performances. Especially, the most paramount prognosticators for advanced osteosarcoma at present were integrated by a novel tool of interactive web dynamic nomogram, making it a more representative and reflective predictor for survival prediction. Simultaneously, compared with previously identified prognostic predictors for osteosarcoma, the features of easy availability, high predictive performances, and conveniences in dynamic monitoring of the two predictive indicators have fully exhibited their superiorities as
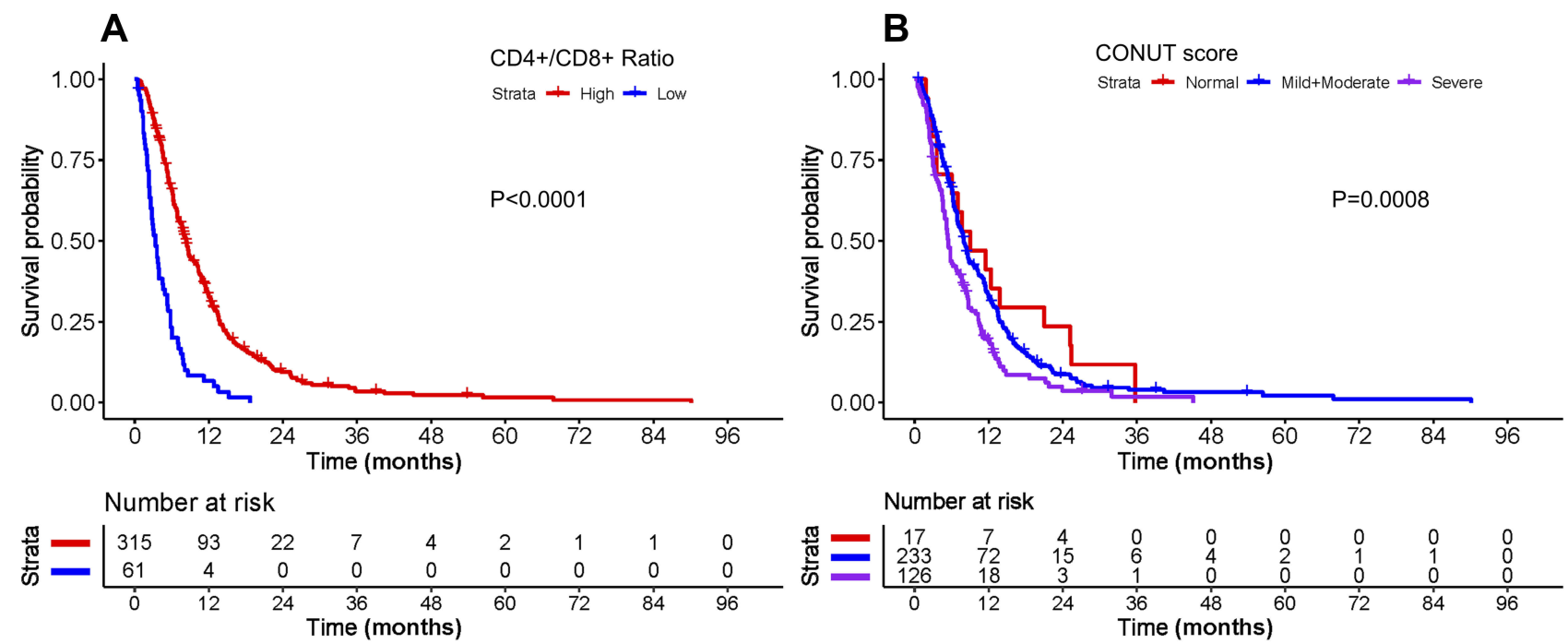

$n=376$

Figure 2 Kaplan-Meier survival curves for overall survival based on (A) CD4+/CD8+ ratio and (B) CONUT score stratification.

Abbreviations: $C D$, cluster of differentiation; CONUT score, controlling nutritional status score. 
Table 4 Parameters of Each Variable Selected for the Three Models

\begin{tabular}{|c|c|c|c|c|c|c|c|c|c|}
\hline \multirow[t]{2}{*}{ Variables } & \multicolumn{3}{|c|}{ Model I } & \multicolumn{3}{|c|}{ Model 2} & \multicolumn{3}{|c|}{ Model 3} \\
\hline & Coef. & $\operatorname{HR}(95 \% \mathrm{Cl})$ & $P$ value & Coef. & $\operatorname{HR}(95 \% \mathrm{Cl})$ & $P$ value & Coef. & $\operatorname{HR}(95 \% \mathrm{Cl})$ & $P$ value \\
\hline Age & 0.380 & $1.463(1.146-1.868)$ & 0.002 & 0.355 & $1.427(1.116-1.825)$ & 0.004 & 0.391 & $1.479(1.157-1.890)$ & 0.001 \\
\hline Metastasis & 0.821 & $2.274(1.769-2.922)$ & 0.000 & 0.832 & $2.299(1.788-2.957)$ & 0.000 & 0.861 & $2.367(1.839-3.048)$ & 0.000 \\
\hline ALP & 0.612 & $1.844(1.330-2.557)$ & 0.000 & 0.576 & $1.779(1.280-2.473)$ & 0.000 & 0.594 & $1.812(1.303-2.519)$ & 0.000 \\
\hline Chemotherapy & 0.489 & I.632(I.199-2.222) & 0.001 & 0.529 & I.698(I.248-2.309) & 0.000 & 0.444 & $1.559(1.142-2.126)$ & 0.005 \\
\hline CD4+/CD8+ ratio & 1.279 & $3.596(2.60 I-4.972)$ & 0.000 & - & - & - & 1.273 & $3.574(2.58 \mid-4.948)$ & 0.000 \\
\hline \multicolumn{10}{|l|}{ CONUT score } \\
\hline Mild+moderate & - & - & - & 0.385 & $|.47|(0.844-2.563)$ & 0.173 & 0.481 & $1.619(0.928-2.824)$ & 0.089 \\
\hline Severe & - & - & - & 0.785 & $2.193(1.230-3.909)$ & 0.007 & 0.827 & $2.287(1.284-4.075)$ & 0.004 \\
\hline AIC & \multicolumn{3}{|c|}{2524.100} & \multicolumn{3}{|c|}{2561.900} & \multicolumn{3}{|l|}{2516.7} \\
\hline
\end{tabular}

Abbreviations: Coef, coefficient; HR, hazard ratio; CD, cluster of differentiation; ALP, alkaline phosphatase; AIC, Akaike information criterion; CONUT score, controlling nutritional status score.

Table 5 Detailed Point of Each Predictor in the Three Established Nomograms

\begin{tabular}{|c|c|c|c|}
\hline Variables & Model I & Model 2 & Model 3 \\
\hline \multicolumn{4}{|l|}{ Age } \\
\hline$<20$ years & 0 & 0 & 0 \\
\hline$\geq 20$ years & 29.744 & 42.707 & 30.735 \\
\hline \multicolumn{4}{|l|}{ Metastasis } \\
\hline Lung only & 0 & 0 & 0 \\
\hline Lung+other sites & 64.180 & 100.000 & 67.666 \\
\hline \multicolumn{4}{|l|}{ ALP } \\
\hline Low & 0 & 0 & 0 \\
\hline High & 47.821 & 69.207 & 46.690 \\
\hline \multicolumn{4}{|l|}{$\mathrm{CD} 4+/ \mathrm{CD} 8+$ ratio } \\
\hline High ( $\geq 3.27)$ & 0 & - & 0 \\
\hline Low $(<3.27)$ & 100.000 & - & 100.000 \\
\hline \multicolumn{4}{|l|}{ Chemotherapy } \\
\hline$\geq 6$ cycles & 0 & 0 & 0 \\
\hline$<6$ cycles & 38.265 & 63.577 & 34.861 \\
\hline \multicolumn{4}{|l|}{ CONUT score } \\
\hline Normal & - & 0 & 0 \\
\hline Mild+moderate & - & 46.334 & 37.831 \\
\hline Severe & - & 94.315 & 64.963 \\
\hline
\end{tabular}

Abbreviations: ALP, alkaline phosphatase; CD, cluster of differentiation; CONUT score, controlling nutritional status score.

pragmatic and cost-effective biomarkers for survival stratification in osteosarcoma.

Nutritional and immune status has been proven to correlate with therapeutic effects and long-term prognosis in various tumors, including osteosarcoma. ${ }^{5,6,20,47}$ A series of nutrition- and immune-based indices or scoring systems were developed and utilized as prognostic predictors, ${ }^{6,48-51}$
Table 6 Variables Included in the Prediction Models and Websites for the Individualized Dynamic Nomograms

\begin{tabular}{|c|c|c|}
\hline Model & Variables & Websites \\
\hline Model I & $\begin{array}{l}\text { (Overall survival, Survival } \\
\text { status) } \sim \text { Age + Metastasis }+ \\
\text { ALP + Chemotherapy + CD4 } \\
\text { +/CD8+ Ratio }\end{array}$ & $\begin{array}{l}\text { https://conquerera.shi } \\
\frac{\text { nyapps.io/ }}{\underline{\text { DynNomapp I/ }}}\end{array}$ \\
\hline Model 2 & $\begin{array}{l}\text { (Overall survival, Survival } \\
\text { status) } \sim \text { Age }+ \text { Metastasis }+ \\
\text { ALP }+ \text { Chemotherapy }+ \\
\text { CONUT Score }\end{array}$ & $\begin{array}{l}\text { https://conquerera.shi } \\
\frac{\text { nyapps.io/ }}{\underline{\text { DynNomapp2/ }}}\end{array}$ \\
\hline Model 3 & $\begin{array}{l}\text { (Overall survival, Survival } \\
\text { status) } \sim \text { Age + Metastasis + } \\
\text { ALP + Chemotherapy + CD4 } \\
\text { +/CD8+ Ratio + CONUT } \\
\text { Score }\end{array}$ & $\begin{array}{l}\text { https://conquerera.shi } \\
\frac{\text { nyapps.io/ }}{\text { DynNomapp3/ }}\end{array}$ \\
\hline
\end{tabular}

Abbreviations: ALP, alkaline phosphatase; CD, cluster of differentiation; CONUT score, controlling nutritional status score.

with the CONUT score being the most commonly used one among them. As an immune-nutritional marker, CONUT score has included the information of serum levels of albumin, cholesterol, and TLC. Albumin, reflecting the protein synthesis ability of liver and forming a large proportion of all plasma protein, is the most abundant blood plasma protein. Serum albumin level is the most fundamental factor to evaluate a patient's nutritional status, and it has been proven to significantly associate with the survival of multiple malignancies. ${ }^{52-55}$ Cholesterol, another vital component of CONUT score, is also a major indicator of nutritional status and represents the lipid metabolism ability as well as a patient's caloric reserves. ${ }^{56}$ By integrating into the 


\section{A (Model1)}

Points

Age

Metastasis

ALP

Chemotherapy

CD4+/CD8+ Ratio

Total Points

Median Survival Time

1-years Survival Probability

3-years Survival Probability

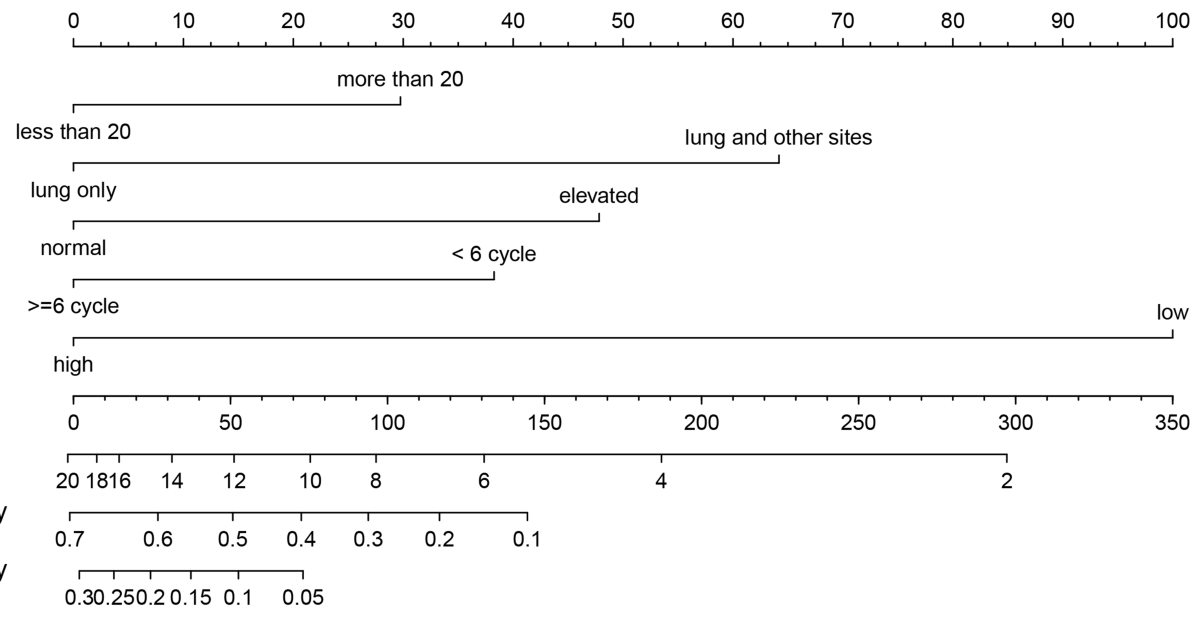

\section{B (Model2)}

Points

Age

Metastasis

ALP

Chemotherapy

CONUT score

Total Points

Median Survival Time

1-years Survival Probability

3-years Survival Probability

\begin{tabular}{|c|c|c|c|c|c|c|c|c|c|c|}
\hline 0 & 10 & 20 & 30 & 40 & 50 & 60 & 70 & 80 & 90 & 100 \\
\hline
\end{tabular}

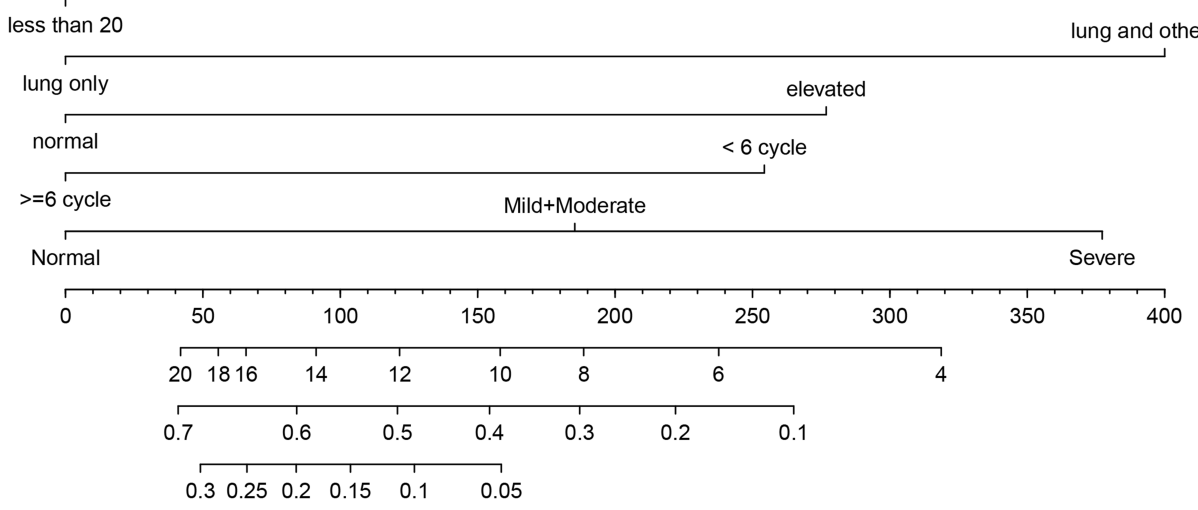

\section{C (Model3)}

Points

Age

Metastasis

ALP

Chemotherapy

CD4+/CD8+ Ratio

CONUT score

Total Points

Median Survival Time

1-years Survival Probability

3-years Survival Probability

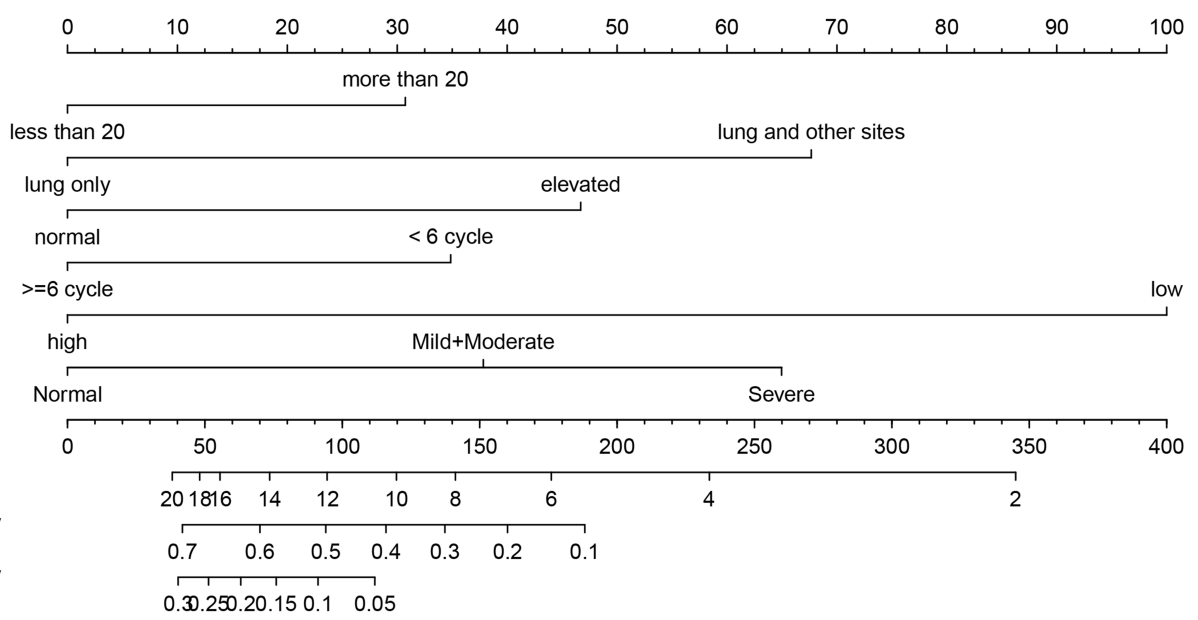

Figure 3 Nomograms of the established three prediction models to predict median survival time, I-, and 3-year survival probabilities. (A) CD4+/CD8+ ratio model (model I); (B) CONUT score model (model 2); (C) combined model of CD4+/CD8+ ratio and CONUT score (model 3).

Abbreviations: ALP, alkaline phosphatase; CD, cluster of differentiation; CONUT score, controlling nutritional status score. 
A

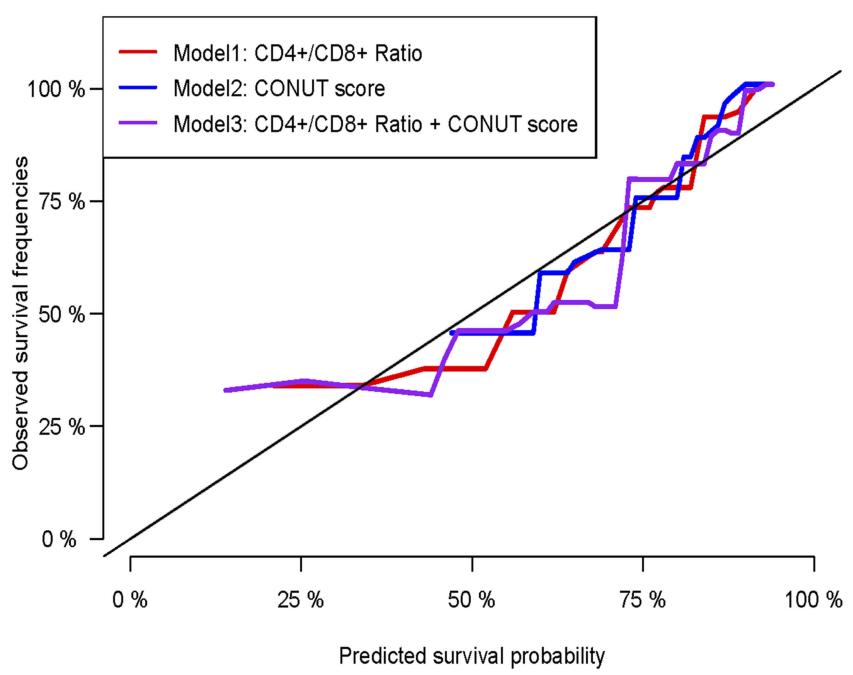

B Validation set

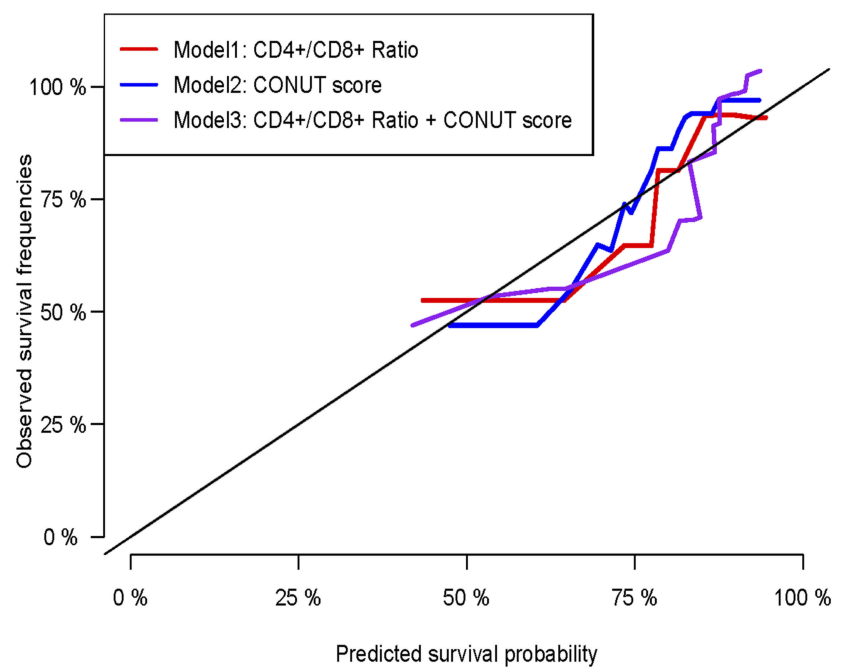

Figure 4 The calibration plots of the established three prediction models to predict I-year survival in the (A) development set and (B) validation set. The $X$-axis represents the predicted survival from the established prognostic models and the $Y$-axis reflects the actual survival in the real world. The color curve closest to the $45^{\circ}$ tilting solid line symbolizes the most accurate prediction model and behave the best in predictive performance.

Abbreviations: CD, cluster of differentiation; CONUT score, controlling nutritional status score.
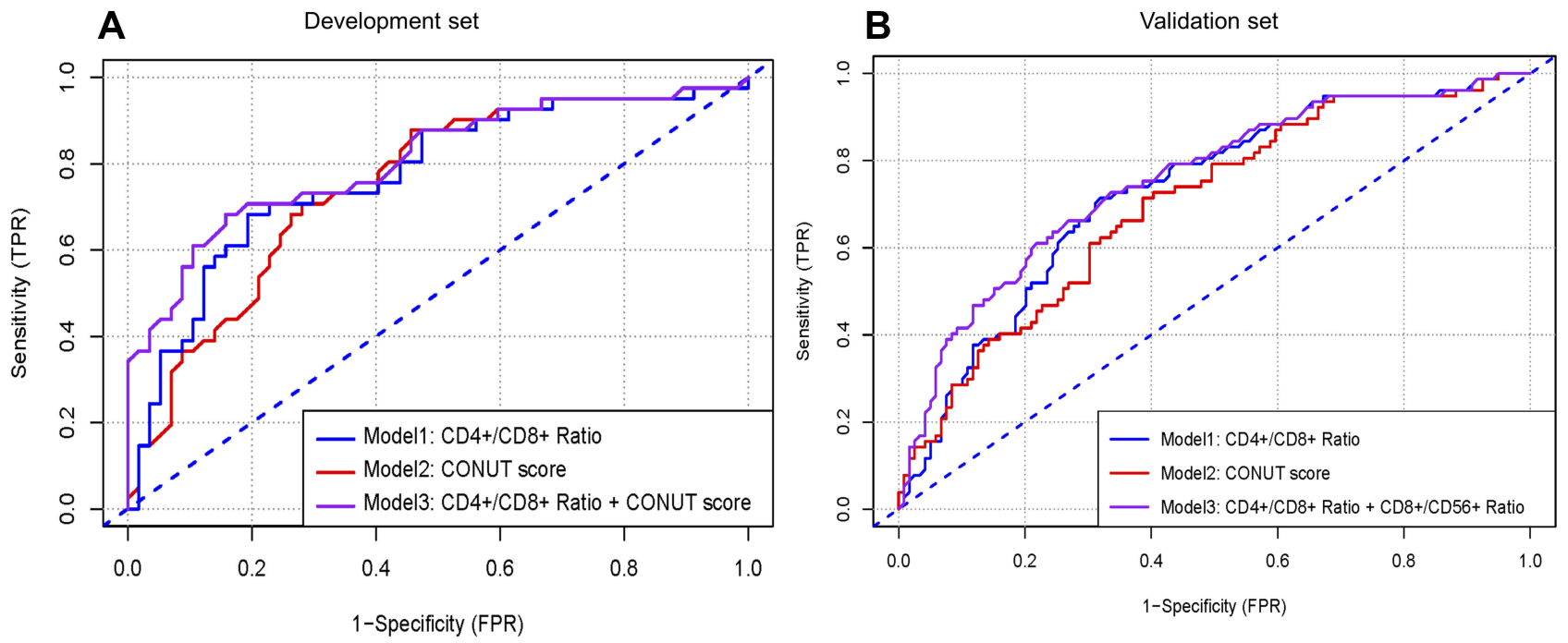

Figure 5 The receiver operating characteristic (ROC) curve analyses of the three prediction models for I-year survival in the (A) development set and (B) validation set. Abbreviations: CD, cluster of differentiation; CONUT score, controlling nutritional status score.

specialized lipid-protein membrane micro-domains, cholesterol forms the signaling transduction machinery and participates in various biological processes of tumor cells, including cytoskeleton alteration, cell polarity, and angiogenesis. ${ }^{57-60}$ Similar to hypoalbuminemia, hypocholesterolemia is also associated with undesirable outcomes in numerous cancers, such as renal cell carcinoma, lung cancer, hematologic malignancies, etc. ${ }^{61-64}$ Furthermore, hypocholesterolemia can cripple the functions of immune cells by disturbing the normal operation of cell membrane fluidity and obstructing cell surface receptors mobility. ${ }^{65}$ It is noteworthy that cholesterol is the main difference between CONUT score and other prognostic markers. It is undeniable that albumin is the most prominent indicator for nutrition, but one obvious drawback for albumin is that its serum concentration can be easily influenced by inadequate nutrients intake, inflammation factor-induced exhaustion, and changes of body fluid volume. By contrast, serum cholesterol circumvents such weaknesses and remains much more stable. Therefore, the inclusion of cholesterol 
Table 7 Accuracy of the Three Models to Predict I-Year Survival Probability in Advanced Osteosarcoma Patients

\begin{tabular}{|l|l|l|l|l|l|l|}
\hline \multirow{2}{*}{ Variable } & \multicolumn{2}{l|}{ Model I } & \multicolumn{2}{l|}{ Model 2 } & \multicolumn{2}{l|}{ Model 3 } \\
\cline { 2 - 7 } & Development Set & Validation Set & Development Set & Validation Set & Development Set & Validation Set \\
\hline Cut-off point & 77.564 & 102.445 & 158.247 & 209.911 & 119.382 & 140.357 \\
AUC & 0.773 & 0.780 & 0.749 & 0.700 & 0.848 & 0.835 \\
Sen (\%) & 0.927 & 0.818 & 0.683 & 0.613 & 0.707 & 0.779 \\
Spe (\%) & 0.526 & 0.689 & 0.807 & 0.714 & 0.895 & 0.756 \\
PV+ (\%) & 94.565 & 93.548 & 93.401 & 93.333 & 93.627 & 94.118 \\
PV- (\%) & 11.965 & 15.909 & 10.577 & 15.556 & 11.340 & 17.073 \\
LR+ & 1.956 & 2.630 & 3.539 & 2.143 & 6.733 & 3.193 \\
LR- & 0.139 & 0.264 & 0.393 & 0.542 & 0.327 & 0.292 \\
\hline
\end{tabular}

Abbreviations: AUC, area under curve; Sen, sensitivity; Spe, specificity; PV+, positive predictive value; PV-, negative predictive value; LR+, positive likelihood ratio; LR-, negative likelihood ratio.

into CONUT score can achieve optimization of this scoring system. The last important constituent parameter of CONUT score is TLC, which is an indicator of a patient's immunocompetence and also a paramount player in antitumor immune response. TLC consists of numerous cell subsets, including CD4 $+\mathrm{T}$ cell, CD8 $+\mathrm{T}$ cell, NK cells, $\gamma \delta$ $\mathrm{T}$ cells, B cells, Tregs, etc. TLC has been reported to be closely related to tumor activities and prognosis. Generally, quantity change or disproportionality of lymphocytes in peripheral blood or TME is a potent predictor for impaired tumor immunity. For example, reduced ratio of CD4+/CD8+ in TME, ${ }^{66}$ low densities of tumor-associated plasma cells, ${ }^{67}$ low ratio of FOXP3+/CD4+ TILs, ${ }^{68}$ and decreased ratio of intratumoral FOXP+ Tregs are all indicative of unfavorable prognosis in patients with neoplasms. ${ }^{69}$ Considering the strong associations between albumin, cholesterol, and TLC and the prognosis in various tumors, it may be reasonable for us to speculate that a multidimensional prognosticator based on these predictors would also obtain optimal performances in survival prediction. Consistent with our anticipation, our study suggested that CONUT score was an independent risk factor for OS and a higher CONUT score was significantly correlated with shorter OS. Furthermore, a prediction model based on CONUT score also obtained favorable predictive performances, including a desirable C-index of $0.712(95 \% \mathrm{CI}$ : $0.738-0.908)$ and 0.723 (95\% CI: $0.667-0.876)$ in the development and validation set, as well as good consistency between nomogram predicted survival and actual observed survival in both sets.

Scarce information can be found in previous studies that focused on elucidating the prognostic values of peripheral
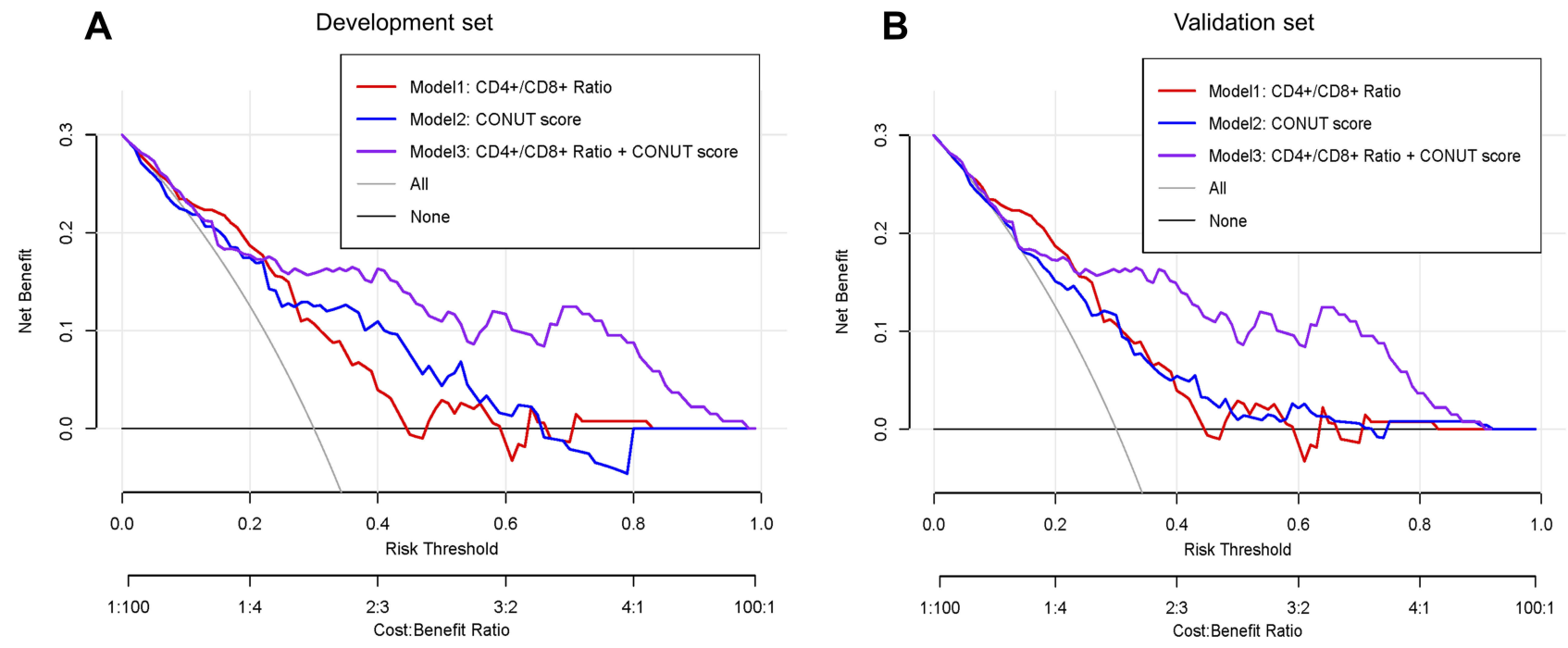

Figure 6 Decision curve analysis of the three prediction models in the (A) development set and (B) validation set. Abbreviations: $C D$, cluster of differentiation; CONUT score, controlling nutritional status score. 

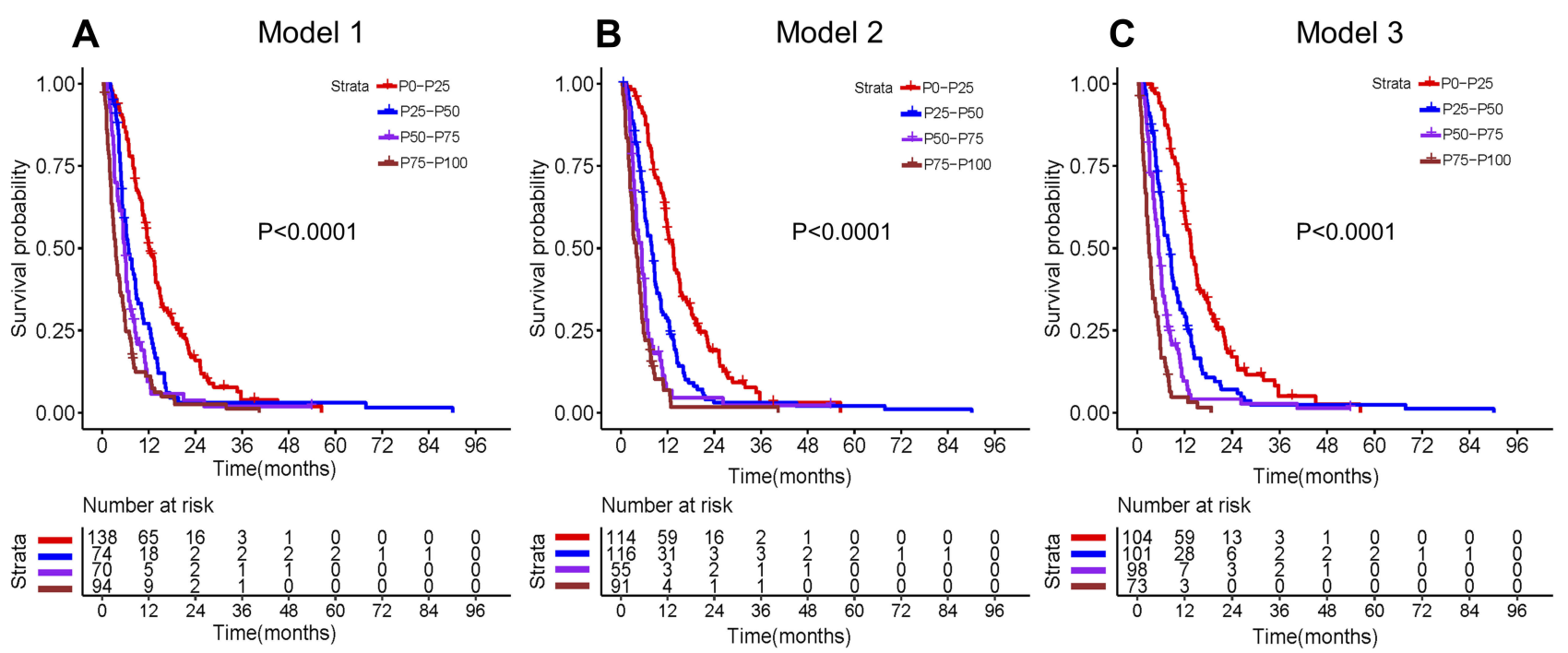

Figure 7 Kaplan-Meier survival curves of the entire cohort stratified by the quartiles of the calculated total prognostic scores from the three prediction models. (A) CD4+/CD8+ ratio model (model I); (B) CONUT score model (model 2); (C) combined model of CD4+/CD8+ ratio and CONUT score (model 3).

Abbreviations: $\mathrm{CD}$, cluster of differentiation; CONUT score, controlling nutritional status score.

blood or intratumoral lymphocyte subsets in sarcomas. Our study was the first to uncover the prognostic significance of peripheral blood CD4+/CD8+ ratio in osteosarcoma. In summary, the prognostic values of lymphocyte subsets for tumors mainly display in three aspects: quantitative changes, disturbance of proportions, and aberrant gene expression profile. Usually, increased circulating or intratumoral immunosuppressive immune cells or decreased immunity-boosting lymphocytes are more prone to result in undesirable OS. Similarly, a ratio of two specific lymphocyte subsets within which anti-tumor lymphocytes predominate over pro-tumor lymphocytes is also more likely to obtain a shorter OS. For example, elevated intratumoral CD163+ M2-type TAM, ${ }^{31}$ lower intratumoral CD8+ cytotoxic lymphocytes, ${ }^{32}$ intratumoral CD8+/FOXP3+-ratio $<3.08,{ }^{1}$ delayed peripheral lymphocyte recovery, ${ }^{28}$ decreased CD8+/Tregs ratio, ${ }^{35} \mathrm{M} 1 / \mathrm{M} 2$ polarized-macrophages tilt to M2 subtype, ${ }^{34}$ low CD4+/CD8 + ratio, ${ }^{38}$ and decreased pre-treatment $\mathrm{TLC}^{70}$ all forebode worse prognosis. In our study, we found that lower peripheral CD4+/CD8+ ratio was indicative of poorer prognosis, and prediction model based on $\mathrm{CD} 4+/ \mathrm{CD} 8+$ ratio and clinical features has revealed good predictive performances for patients with advanced osteosarcoma. Noteworthily, although CONUT score has taken TLC into consideration to reflect a patient's immune status, the information obtained from TLC is rather rough, inadequate, and imprecise for the reason that TLC is composed of various cell subsets. Given this, the information from the overall change of TLC provided by CONUT score is rather limited. To guide clinical decisions, more elaborate information about the dynamic variations of lymphocyte subsets is thus needed. In this context, we attempt to integrate CONUT score and peripheral CD4+/CD8+ ratio together to establish a new prognostic prediction model. Fortunately, the combined model (model 3) of CONUT score, peripheral CD4+/CD8+ ratio, and clinical parameters behaves better in prediction performances than prediction models based on CONUT score or peripheral $\mathrm{CD} 4+/ \mathrm{CD} 8+$ ratio only, which can be deduced from the following facts. Firstly, the combined model gets admirable accuracy in calibration plots when compared with that from the other two prediction models. Secondly, the combined model has reached higher AUCs of 0.848 and 0.835 in the development set and validation set as opposed to relatively lower AUCs of 0.773 and 0.780 in model 1, and 0.749 and 0.700 in model 2 , respectively. Thirdly, the combined model has shown better ability in risk stratification based on the quartiles of total prediction scores calculated from the established prediction models. Finally, model 3 obtains better net benefit than the other two models within the same threshold probabilities of $36-80 \%$. All these facts mentioned above suggested that CONUT score combined with peripheral $\mathrm{CD} 4+/ \mathrm{CD} 8+$ ratio was an ideal prognostic biomarker for survival prediction in patients with advanced osteosarcoma.

The precise mechanisms for the negative correlation of lower $\mathrm{CD} 4+/ \mathrm{CD} 8+$ ratio and unfavorable OS in osteosarcoma remain uncertain, but preclinical and clinical evidences from other malignancies may account for this phenomenon. 
Generally, except for the engagement of tumor neo-antigen and T-cell receptor, the initial activation of CD8+ cytotoxic lymphocytes induced anti-tumor immune response is also highly depended on the co-stimulatory signaling from CD4 + type 1 helper T cells (Th1). In other words, CD4+ T-cell help initiates and amplifies signals for primary CD8+ T-cell immunity via multiple ways, such as secretion of interleukin2, expression of co-stimulating molecules $\mathrm{B} 7$, production of interleukin-12 and interferon- $\gamma$, and so on. ${ }^{71}$ Therefore, peripheral and intratumoral ratio of $\mathrm{CD} 4+/ \mathrm{CD} 8+\mathrm{T}$ cells, as a surrogate marker of immunosenescence, may independently predict OS in patients with malignant diseases. Depletion or decrease of peripheral and intratumoral CD4+ $\mathrm{T}$ cells is more prone to inactivate the anti-tumor response and lead to deterioration and progression of the disease. Actually, various previous studies have already elucidated and verified that $\mathrm{CD} 4+/ \mathrm{CD} 8+$ ratio can serve as independent prognostic predictors in multiple cancers, including laryngeal squamous cell carcinoma, ${ }^{45}$ renal cell cancer, ${ }^{72}$ nasopharyngeal carcinoma, ${ }^{46}$ gastric cancer, ${ }^{73}$ cervical cancer, ${ }^{74}$ etc. Similarly, our study also demonstrated that CD4+/CD8+ ratio is a reliable and accurate prognostic predictor for patients with advanced osteosarcoma.

Our study also exists several limitations. Firstly, due to the nature of retrospective analysis and single-institution study, a kind of selection bias may be inevitable. So, prospective and larger studies to further verify the generalizability of the established prognostic models in this study are necessarily needed. Secondly, although the relationship between peripheral blood CD4+/CD8+ ratio and survival in osteosarcoma has been clarified in this study, the association of intratumoral $\mathrm{CD} 4+/ \mathrm{CD} 8+$ ratio and survival still remains unclear. Thirdly, despite the advantages of easy availability and convenience in dynamic monitoring for CONUT score and peripheral $\mathrm{CD} 4+/ \mathrm{CD} 8+$ ratio, it is undeniable that the two predictors are all non-specific predictive factors for osteosarcoma, which may unavoidably lose a partial of specificity in survival prediction. One possible way to improve the specificity is to incorporate some newly-identified specific biomarkers into these prognostic prediction models, such as tissue-specific mark proteins, miRNAs, IncRNAs, or circRNAs, but the feasibility still needs further verification.

In conclusion, CONUT score and peripheral CD4+/CD8+ ratio are easily available, reliable, and economical prognostic predictors for survival prediction and stratification in patients with advanced osteosarcoma, but the two predictors combined can establish a better prognosis prediction model.
Simultaneously, the newly developed interactive web dynamic nomogram based on CONUT score and peripheral $\mathrm{CD} 4+/ \mathrm{CD} 8+$ ratio is a more convenient and practical tool for clinicians to perform individualized treatment decisionmaking and evaluate survival at the initial diagnosis.

\section{Abbreviations}

AIC, Akaike information criterion; ALC, absolute lymphocyte count; ALP, alkaline phosphatase; C-index, concordance index; circRNAs, circular RNAs; CONUT score, controlling nutritional status score; DCA, decision curve analysis; EDTA, ethylenediaminetetraacetic acid; FACS, fluorescent activated cell sorting; LMR, lymphocytemonocyte ratio; lncRNAs, long non-coding RNAs; LR-, negative likelihood ratio; $\mathrm{LR}+$, positive likelihood ratio; McAb, monoclonal antibodies; MDT, multidisciplinary therapy; miRNAs, micro-RNAs; MST, median survival time; NK cell, natural killer cell; NLR, neutrophil-lymphocyte ratio; OS, overall survival; PLR, platelet-lymphocyte ratio; $\mathrm{PV}-$, negative predictive value; $\mathrm{PV}+$, positive predictive value; RBC, red blood cell; Sen, sensitivity; Spe, specificity; TAMs, tumor-associated macrophages; TILs, tumor-infiltrating lymphocytes; TLC, total lymphocyte counts; TME, tumor microenvironment; Tregs, Tregulatory cells; VIF, variance inflation factor.

\section{Data Sharing Statement}

The datasets to replicate the results of this study are available from the corresponding author only on reasonable request.

\section{Ethics Approval and Consent to Participate}

The present study was performed in accordance with the World Medical Association Declaration of Helsinki and was approved by the Ethics Committee of Cancer Hospital of China Medical University. This study was a retrospective study and all the data used for the final statistical analysis was anonymous. Therefore, informed consent from the enrolled patients was waived. The institutional review board of our hospital gave ethical approval for this study.

\section{Acknowledgments}

The authors thank Dr Chen Tong from the Department of Bone and Soft Tissue Surgery at Cancer Hospital of China Medical University for providing assistance in data 
collection and statistical analyses. We also thank KGSupport (http://www.kgsupport.com/) for English language editing. This work was supported by grants from the National Natural Science Foundation of China (No. 31371147).

\section{Disclosure}

The authors have no conflicts of interest to disclose in this work.

\section{References}

1. Fritzsching B, Fellenberg J, Moskovszky L, et al. CD8(+)/FOXP3(+)ratio in osteosarcoma microenvironment separates survivors from non-survivors: a multicenter validated retrospective study. Oncoimmunology. 2015;4(3):e990800. doi:10.4161/2162402X.2014. 990800

2. Bacci G, Ferrari S, Bertoni F, et al. Long-term outcome for patients with nonmetastatic osteosarcoma of the extremity treated at the istituto ortopedico rizzoli according to the istituto ortopedico rizzoli/osteosarcoma-2 protocol: an updated report. J Clin Oncol. 2000;18(24):4016-4027. doi:10.1200/JCO.2000.18.24.4016

3. Isakoff MS, Bielack SS, Meltzer P, et al. Osteosarcoma: current treatment and a collaborative pathway to success. J Clin Oncol. 2015;33(27):3029-3035. doi:10.1200/JCO.2014.59.4895

4. Harris MB, Gieser P, Goorin AM, et al. Treatment of metastatic osteosarcoma at diagnosis: a pediatric oncology group study. $J$ Clin Oncol. 1998;16(11):3641-3648. doi:10.1200/JCO.1998.16.11.3641

5. Tenardi RD, Frühwald MC, Jürgens H, et al. Nutritional status of children and young adults with Ewing sarcoma or osteosarcoma at diagnosis and during multimodality therapy. Ped Blood Cancer. 2013;60(1):166. doi:10.1002/pbc.24304

6. Huang $\mathrm{X}, \mathrm{Hu} \mathrm{H}$, Zhang $\mathrm{W}$, et al. Prognostic value of prognostic nutritional index and systemic immune-inflammation index in patients with osteosarcoma. J Cell Physiol. 2019;234(10):1840 8-18414. doi:10.1002/jcp.28476

7. Liu B, Huang Y, Sun Y, et al. Prognostic value of inflammation-based scores in patients with osteosarcoma. Sci Rep. 2016;6:39862. doi:10.1038/srep39862

8. Chui MH, Kandel RA, Wong M, et al. Histopathologic Features Of Prognostic Significance In High-Grade Osteosarcoma. Arch Pathol Lab Med. 2016;140(11):1231-1242. doi:10.5858/arpa.2015-0389-OA

9. Ren L, Hong ES, Mendoza A, et al. Metabolomics uncovers a link between inositol metabolism and osteosarcoma metastasis. Oncotarget. 2017;8(24):38541-38553. doi:10.18632/oncotarget.15872

10. Zhang Z, Qiu Y, Hua Y, et al. Serum and urinary metabonomic study of human osteosarcoma. J Proteome Res. 2010;9(9):4861-4868. doi:10.1021/pr100480r

11. Shi JH, Hao YJ. DDX10 overexpression predicts worse prognosis in osteosarcoma and its deletion prohibits cell activities modulated by MAPK pathway. Biochem Biophys Res Commun. 2019;510 (4):525-529. doi:10.1016/j.bbrc.2019.01.114

12. Berlanga $\mathrm{P}, \mathrm{Mu} \mathrm{OL}$, Piqueras $\mathrm{M}$, et al. miR-200c and phospho-AKT as prognostic factors and mediators of osteosarcoma progression and lung metastasis. Mol Oncol. 2016;10(7):1043-1053. doi:10.1016/j. molonc.2016.04.004

13. Zhang Y, Meng W, Cui H. LncRNA CBR3-AS1 predicts unfavorable prognosis and promotes tumorigenesis in osteosarcoma. Biomed Pharmacother. 2018;102:169-174. doi:10.1016/j.biopha.2018.02.081

14. Nie WB, Zhao LM, Guo R, et al. Circular RNA circ-NT5C2 acts as a potential novel biomarker for prognosis of osteosarcoma. Eur Rev Med Pharmco. 2018;22(19):6239-6244. doi:10.26355/eurrev_2018 10_16030
15. Gonda K, Shibata M, Sato Y, et al. Elevated neutrophil-tolymphocyte ratio is associated with nutritional impairment, immune suppression, resistance to S-1 plus cisplatin, and poor prognosis in patients with stage IV gastric cancer. Mol Clin Oncol. 2017;7 (6):1073-1078. doi:10.3892/mco.2017.1438

16. van Bokhorst-de VDSM, von Blomberg-van DFB, Riezebos RK, et al. Differences in immune status between well-nourished and malnourished head and neck cancer patients. Clin Nutri. 1998;17 (3):107-111. doi:10.1016/s0261-5614(98)80003-4

17. Omura Y, Toiyama Y, Okugawa Y, et al. Crohn's-like lymphoid reaction is associated with oncological prognosis and nutritional status in patients with pathological Stage II/III gastric cancer. Ann Surg Oncol. 2020;27(1):259-267. doi:10.1245/s10434-019-07709-w

18. Tobberup R, Thoresen L, Falkmer UG, et al. Effects of current parenteral nutrition treatment on health-related quality of life, physical function, nutritional status, survival and adverse events exclusively in patients with advanced cancer: A systematic literature review. Crit Rev Oncol Hematol. 2019;139:96-107. doi:10.1016/j. critrevonc.2019.04.014

19. Kirov KM, Xu HP, Crenn P, et al. Role of nutritional status in the early postoperative prognosis of patients operated for retroperitoneal liposarcoma (RLS): a single center experience. Eur J Surg Oncol. 2019;45(2):261-267. doi:10.1016/j.ejso.2018.07.001

20. Takagi K, Umeda Y, Yoshida R, et al. Preoperative controlling nutritional status score predicts mortality after hepatectomy for hepatocellular carcinoma. Digestive Surgery. 2019;36(3):226-232. doi:10. $1159 / 000488215$

21. Kang HW, Seo SP, Kim WT, et al. Prognostic impact of nutritional status assessed by the Controlling Nutritional Status (CONUT) score in patients with surgically treated renal cell carcinoma. Nutri Cancer. 2018;70(6):886-894. doi:10.1080/01635581.2018.1490448

22. Harimoto N, Yoshizumi T, Inokuchi S, et al. Prognostic significance of preoperative Controlling Nutritional Status (CONUT) score in patients undergoing hepatic resection for hepatocellular carcinoma: a multi-institutional study. Ann Surg Oncol. 2018;25(11):3316-3323. doi:10.1245/s10434-018-6672-6

23. Kuroda D, Sawayama H, Kurashige J, et al. Controlling Nutritional Status (CONUT) score is a prognostic marker for gastric cancer patients after curative resection. Gastric Cancer. 2018;21(2):20 4-212. doi:10.1007/s10120-017-0744-3

24. Takagi K, Yagi T, Umeda Y, et al. Preoperative Controlling Nutritional Status (CONUT) score for assessment of prognosis following hepatectomy for hepatocellular carcinoma. World Journal of Surgery. 2017;41(9):2353-2360. doi:10.1007/s00268-017-3985-8

25. Liu T, Fang XC, Ding $Z$, et al. Pre-operative lymphocyte-tomonocyte ratio as a predictor of overall survival in patients suffering from osteosarcoma. FEBS Open Bio. 2015;5:682-687. doi:10.1016/j. fob.2015.08.002

26. Xia WK, Liu ZL, Shen D, et al. Prognostic performance of pre-treatment NLR and PLR in patients suffering from osteosarcoma. World Journal of Surgical Oncology. 2016;14:127. doi:10.1186/s12957-016-0889-2

27. Vasquez L, Leon E, Beltran B, et al. Pretreatment neutrophil-tolymphocyte ratio and lymphocyte recovery: independent prognostic factors for survival in pediatric sarcomas. J Pediatr Hematol Oncol. 2017;39(7):538-546. doi:10.1097/MPH.0000000000000911

28. De Angulo G, Hernandez M, Morales-Arias J, et al. Early lymphocyte recovery as a prognostic indicator for high-risk Ewing sarcoma. $J$ Pediatr Hematol Oncol. 2007;29(1):48-52. doi:10.1097/MPH.0b $013 \mathrm{e} 31802 \mathrm{~d} 3 \mathrm{e} 3 \mathrm{e}$

29. Machado I, Lopez-Guerrero JA, Scotlandi K, et al. Immunohistochemical analysis and prognostic significance of PDL1, PD-1, and CD8+ tumor-infiltrating lymphocytes in Ewing's sarcoma family of tumors (ESFT). Virchows Archiv. 2018;472 (5):815-824. doi:10.1007/s00428-018-2316-2 
30. Heymann MF, Lezot F, Heymann D. The contribution of immune infiltrates and the local microenvironment in the pathogenesis of osteosarcoma. Cellular Immunology. 2019;343:103711. doi:10.1016/ j.cellimm.2017.10.011

31. Han Q, Shi H, Liu F. CD163(+) M2-type tumor-associated macrophage support the suppression of tumor-infiltrating $\mathrm{T}$ cells in osteosarcoma. Int Immunopharmacol. 2016;34:101-106. doi:10.10 16/j.intimp.2016.01.023

32. Gomez-Brouchet A, Illac C, Gilhodes J, et al. CD163-positive tumor-associated macrophages and CD8-positive cytotoxic lymphocytes are powerful diagnostic markers for the therapeutic stratification of osteosarcoma patients: an immunohistochemical analysis of the biopsies from the French OS2006 Phase 3 trial. oncoimmunology. 2017;6(9):e1331193. doi:10.1080/2162402X.2017.1331193

33. Buddingh EP, Kuijjer ML, Duim RA, et al. Tumor-infiltrating macrophages are associated with metastasis suppression in high-grade osteosarcoma: a rationale for treatment with macrophage activating agents. Clin Cancer Res. 2011;17(8):2110-2119. doi:10.1158/10780432.CCR-10-2047

34. Dumars C, Ngyuen JM, Gaultier A, et al. Dysregulation of macrophage polarization is associated with the metastatic process in osteosarcoma. Oncotarget. 2016;7(48):78343-78354. doi:10.18632/ oncotarget. 13055

35. Biller BJ, Guth A, Burton JH, et al. Decreased ratio of CD8+ T cells to regulatory $\mathrm{T}$ cells associated with decreased survival in dogs with osteosarcoma. J Veterinary Intern Med. 2010;24(5):1118-1123. doi:10.1111/j.1939-1676.2010.0557.x

36. Sevcikova L, Hunakova L, Chorvath B, et al. T-lymphocyte subsets (CD4/CD8 ratio) in breast cancer patients. Neoplasma. 1992;39 (4):219-222. PMID: 1436231.

37. Hernberg M, Muhonen T, Pyrhonen S. Can the CD4+/CD $8+$ ratio predict the outcome of interferon-alpha therapy for renal cell carcinoma? Ann Oncol. 1997;8(1):71-77. doi:10.1023/a:1008293117223

38. Hernberg M, Muhonen T, Turunen JP, et al. The CD4+/CD8+ ratio as a prognostic factor in patients with metastatic melanoma receiving chemoimmunotherapy. J Clin Oncol. 1996;14(5):1690-1696. doi:10. 1023/a:1008293117223

39. Hoesli R, Birkeland AC, Rosko AJ, et al. Proportion of CD4 and CD8 tumor infiltrating lymphocytes predicts survival in persistent/ recurrent laryngeal squamous cell carcinoma. Oral Oncol. 2018;77:83-89. doi:10.1016/j.oraloncology.2017.12.003

40. Ohba T, Takamori S, Toyozawa R, et al. Prognostic impact of the Controlling Nutritional Status score in patients with non-small cell lung cancer treated with pembrolizumab. J Thor Dis. 2019;11 (9):3757-3768. doi:10.21037/jtd.2019.09.29

41. Takagi K, Domagala P, Polak WG, et al. Prognostic significance of the controlling nutritional status (CONUT) score in patients undergoing gastrectomy for gastric cancer: a systematic review and meta-analysis. BMC Surg. 2019;19(1):129. doi:10.1186/s12893-0190593-6

42. $\mathrm{Xu} \mathrm{H}$, Tan $\mathrm{P}$, Jin $\mathrm{X}$, et al. Validation of the preoperative controlling nutritional status score as an independent predictor in a large Chinese cohort of patients with upper tract urothelial carcinoma. Cancer Med. 2018;7(12):6112-6123. doi:10.1002/cam4.1902

43. Sun X, Luo L, Zhao X, Ye P. Controlling Nutritional Status (CONUT) score as a predictor of all-cause mortality in elderly hypertensive patients: a prospective follow-up study. BMJ Open. 2017;7(9):e15649. doi:10.1136/bmjopen-2016-015649

44. Hu FJ, Ge MH, Li P, et al. Unfavorable clinical implications of circulating CD44+ lymphocytes in patients with nasopharyngeal carcinoma undergoing radiochemotherapy. Clin Chim Acta. 2012;413 (1-2):213-218. doi:10.1016/j.cca.2011.09.026

45. Zhang D, Tang WJ, Tang D, et al. The ratio of CD4/CD8 T-cells in human papillomavirus-positive laryngeal squamous cell carcinoma accounts for improved outcome. Acta Oto-Laryngologica. 2016;136 (8):826-833. doi:10.3109/00016489.2016.1164341
46. Tao CJ, Chen YY, Jiang F, et al. A prognostic model combining CD4/ $\mathrm{CD} 8$ ratio and $\mathrm{N}$ stage predicts the risk of distant metastasis for patients with nasopharyngeal carcinoma treated by intensity modulated radiotherapy. Oncotarget. 2016;7(29):46653-46661. doi:10.18 632/oncotarget.9695

47. Loan B, Nakahara S, Tho BA, et al. Nutritional status and postoperative outcomes in patients with gastrointestinal cancer in Vietnam: a retrospective cohort study. Nutrition (Burbank, Los Angeles County, Calif). 2018;48:117-121. doi:10.1016/j.nut.2017. 11.027

48. $\mathrm{Hu} \mathrm{S}$, Chen $\mathrm{L}$, Lin $\mathrm{C}$, et al. The prognostic value of preoperative geriatric nutritional risk index in patients with pancreatic ductal adenocarcinoma. Cancer Manag Res. 2020;12:385-395. doi:10.21 47/CMAR.S229341

49. Li YJ, Yao K, Lu MX, et al. Prognostic value of the C-reactive protein to albumin ratio: a novel inflammation-based prognostic indicator in osteosarcoma. Onco Targets Ther. 2017;10:5255-5261. doi:10.2147/OTT.S140560

50. Nakagawa N, Yamada S, Sonohara F, et al. Clinical implications of naples prognostic score in patients with resected pancreatic cancer. Ann Surg Oncol. 2020;27(3):887-895. doi:10.1245/s10434-019-08 047-7

51. Galizia G, Auricchio A, de Vita F, et al. Inflammatory and nutritional status is a predictor of long-term outcome in patients undergoing surgery for gastric cancer. Validation of the Naples prognostic score. Ann Ital Chir. 2019;90:404-416. PMID: 31814602.

52. Gupta D, Lis CG. Pretreatment serum albumin as a predictor of cancer survival: a systematic review of the epidemiological literature. Nutri J. 2010;9:69. doi:10.1186/1475-2891-9-69

53. Willegger M, Posch F, Schieder S, et al. Serum creatinine and albumin predict sarcoma-specific survival in patients with myofibroblastic and fibroblastic sarcomas. J Orthopaedic Res. 2017;35 (12):2815-2824. doi:10.1002/jor.23598

54. Zhang J, Liu X, Yang Z, et al. The pretreatment albumin to globulin ratio, a validated biomarker, predicts prognosis in hepatocellular carcinoma. J Buon. 2016;21(4):925-934. PMID: 27685915.

55. Cong $\mathrm{L}, \mathrm{Hu} \mathrm{L}$. The value of the combination of hemoglobin, albumin, lymphocyte and platelet in predicting platinum-based chemoradiotherapy response in male patients with esophageal squamous cell carcinoma. Int Immunopharmacol. 2017;46:75-79. doi:10.1016/j. intimp.2017.02.027

56. Gadgil MD, Anderson CA, Kandula NR, et al. Dietary patterns are associated with metabolic risk factors in South Asians living in the United States. J Nutri. 2015;145(6):1211-1217. doi:10.3945/jn.114. 207753

57. Lee BH, Taylor MG, Robinet $\mathrm{P}$, et al. Dysregulation of cholesterol homeostasis in human prostate cancer through loss of ABCA1. Cancer Res. 2013;73(3):1211-1218. doi:10.1158/0008-5472.CAN12-3128

58. Morales A, Mari M, Garcia-Ruiz C, et al. Hepatocarcinogenesis and ceramide/cholesterol metabolism. Anti Cancer Agents Med Chem. 2012;12(4):364-375. doi:10.2174/187152012800228689

59. Mandal CC, Rahman MM. Targeting intracellular cholesterol is a novel therapeutic strategy for cancer treatment. J Cancer Sci Ther. 2014;6(12):510-513. doi:10.4172/1948-5956.1000316

60. Nelson ER, Chang C, McDonnell DP. Cholesterol and breast cancer pathophysiology. Trends Endocrinol Metab. 2014;25(12):649-655. doi:10.1016/j.tem.2014.10.001

61. Muller CP, Wagner AU, Maucher C, et al. Hypocholesterolemia, an unfavorable feature of prognostic value in chronic myeloid leukemia. Eur J Haematol. 1989;43(3):235-239. doi:10.1111/j.1600-0609.1989. tb00288.x

62. Sok M, Ravnik J, Ravnik M. Preoperative total serum cholesterol as a prognostic factor for survival in patients with resectable non-smallcell lung cancer. Wiener klinische Wochenschrift. 2009;121(9-10):314-317. doi:10.1007/s00508-009-1169-8 
63. Cucuianu A, Malide D, Petrov L, et al. Serum cholesterol and apoprotein B levels and serum cholinesterase activity in selected hematologic malignancies. ROM J Intern Med. 1992;30(4):261-268. PMID: 1299417.

64. de Martino M, Leitner CV, Seemann C, et al. Preoperative serum cholesterol is an independent prognostic factor for patients with renal cell carcinoma (RCC). BJU Int. 2015;115(3):397-404. doi:10.1590/ S1677-5538.IBJU.2019.0560

65. Gombos I, Kiss E, Detre C, et al. Cholesterol and sphingolipids as lipid organizers of the immune cells' plasma membrane: their impact on the functions of MHC molecules, effector T-lymphocytes and T-cell death. Immunol Lett. 2006;104(1-2):59-69. doi:10.1016/j. imlet.2005.11.021

66. Das D, Sarkar B, Mukhopadhyay S, et al. An Altered Ratio of CD4+ And CD8+ T lymphocytes in cervical cancer tissues and peripheral blood - a prognostic clue? Asian Pac J Cancer Prev. 2018;19 (2):471-478. DOI:10.22034/APJCP.2018.19.2.471

67. Yeong J, Lim J, Lee B, et al. High densities of tumor-associated plasma cells predict improved prognosis in triple negative breast cancer. Front Immunol. 2018;9:1209. doi:10.3389/fimmu.2018.01209

68. Droeser R, Zlobec I, Kilic E, et al. Differential pattern and prognostic significance of $\mathrm{CD} 4+$, FOXP3+ and IL-17+ tumor infiltrating lymphocytes in ductal and lobular breast cancers. BMC Cancer. 2012;12:134. doi:10.1186/1471-2407-12-134
69. Hanke T, Melling N, Simon R, et al. High intratumoral FOXP3(+) $\mathrm{T}$ regulatory cell (Tregs) density is an independent good prognosticator in nodal negative colorectal cancer. Int J Clin Exp Pathol. 2015;8(7):8227-8235. PMID: 26339391.

70. Suzuki R, Lin SH, Wei X, et al. Prognostic significance of pretreatment total lymphocyte count and neutrophil-to-lymphocyte ratio in extensive-stage small-cell lung cancer. Radiother Oncol. 2018;126 (3):499-505. doi:10.1016/j.radonc.2017.12.030

71. Bedoui S, Heath WR, Mueller SN. CD4 +T-cell help amplifies innate signals for primary CD8+T-cell immunity. Immunol Rev. 2016;272 (1):52-64. doi:10.1111/imr.12426

72. Nishida K, Kawashima A, Kanazawa T, et al. Clinical importance of the expression of CD4+CD8+ $\mathrm{T}$ cells in Renal Cell Carcinoma. Int Immunol. 2020;32(5):347-357. doi:10.1093/intimm/dxaa004

73. Li F, Sun Y, Huang J, et al. CD4/CD8 + T cells, DC subsets, Foxp3, and IDO expression are predictive indictors of gastric cancer prognosis. Cancer Med. 2019;8(17):7330-7344. doi:10.1002/cam4. 2596

74. Shah W, Yan X, Jing L, et al. A reversed CD4/CD8 ratio of tumor-infiltrating lymphocytes and a high percentage of CD4(+) FOXP3 $(+)$ regulatory T cells are significantly associated with clinical outcome in squamous cell carcinoma of the cervix. Cellular \& Molecular Immunology. 2011;8(1):59-66. doi:10.1038/cmi.2010.56

\section{Publish your work in this journal}

Cancer Management and Research is an international, peer-reviewed open access journal focusing on cancer research and the optimal use of preventative and integrated treatment interventions to achieve improved outcomes, enhanced survival and quality of life for the cancer patient.
The manuscript management system is completely online and includes a very quick and fair peer-review system, which is all easy to use. Visit http://www.dovepress.com/testimonials.php to read real quotes from published authors. 